\title{
Optimization of the Split-Step Fourier Method in Modeling Optical-Fiber Communications Systems
}

\author{
Oleg V. Sinkin, Member, IEEE, Ronald Holzlöhner, Student Member, IEEE, John Zweck, and \\ Curtis R. Menyuk, Fellow, IEEE, Fellow, OSA
}

\begin{abstract}
We studied the efficiency of different implementations of the split-step Fourier method for solving the nonlinear Schrödinger equation that employ different step-size selection criteria. We compared the performance of the different implementations for a variety of pulse formats and systems, including higher order solitons, collisions of soliton pulses, a single-channel periodically stationary dispersion-managed soliton system, and chirped return to zero systems with single and multiple channels. We introduce a globally third-order accurate split-step scheme, in which a bound on the local error is used to select the step size. In many cases, this method is the most efficient when compared with commonly used step-size selection criteria, and it is robust for a wide range of systems providing a system-independent rule for choosing the step sizes. We find that a step-size selection method based on limiting the nonlinear phase rotation of each step is not efficient for many optical-fiber transmission systems, although it works well for solitons. We also tested a method that uses a logarithmic step-size distribution to bound the amount of spurious four-wave mixing. This method is as efficient as other second-order schemes in the single-channel dispersion-managed soliton system, while it is not efficient in other cases including multichannel simulations. We find that in most cases, the simple approach in which the step size is held constant is the least efficient of all the methods. Finally, we implemented a method in which the step size is inversely proportional to the largest group velocity difference between channels. This scheme performs best in multichannel optical communications systems for the values of accuracy typically required in most transmission simulations.
\end{abstract}

Index Terms-Adaptive algorithms, numerical analysis, optical fiber communication simulation, optical propagation, optical solitons, software peformance, split-step Fourier method (SSFM), time-frequency analysis.

\section{INTRODUCTION}

$\mathbf{T}$ HE NONLINEAR Schrödinger equation, which can be written as

$$
i \frac{\partial u}{\partial z}-\frac{\beta^{\prime \prime}}{2} \frac{\partial^{2} u}{\partial t^{2}}+\gamma|u|^{2} u=0
$$

has been shown to govern the propagation of light in a lossless optical fiber with second-order dispersion [1]. In (1), $u$ is the complex field envelope, $z$ is distance, $\beta^{\prime \prime}$ is the second-order dispersion, and $\gamma$ is the nonlinear coefficient. The quantity $t=$

Manuscript received December 17, 2001; revised August 13, 2002. This work was supported in part by the National Science Foundation and the Department of Energy.

O. V. Sinkin, R. Holzlöhner, and J. Zweck are with the Department of Computer Science and Electrical Engineering, University of Maryland Baltimore County, Baltimore, MD 21250 USA (e-mail: osinki1 @umbc.edu).

C. R. Menyuk is with the Department of Computer Science and Electrical Engineering, University of Maryland Baltimore County, Baltimore, MD 21250 USA, and is also with the PhotonEx Corporation, Maynard, MA 01754 USA.

Digital Object Identifier 10.1109/JLT.2003.808628 $\tau-z / v_{g}$ is the retarded time, where $\tau$ is physical time and $v_{g}$ is the group velocity. Even though (1) does not provide a complete physical description of a system, it is the basis for modeling optical-fiber communications systems. Indeed, one can modify the nonlinear Schrödinger equation (1) to incorporate the effects of fiber loss, third-order dispersion, amplification, amplified spontaneous emission noise, and polarization mode dispersion to obtain a more realistic model of optical-fiber transmission [2]-[4]. Equation (1) with $\beta^{\prime \prime}<0$ has a well-known analytical soliton solution with a set of remarkable properties, which gives deep insight into the nature of the dispersive and nonlinear effects [5]. However, in almost all cases, (1) and its modifications cannot be solved analytically and one has to use numerical approaches. The most commonly used numerical scheme for solving (1) is the split-step Fourier method, which is convenient for its simplicity and flexibility in dealing with higher order dispersion, the Raman effect, and filtering [2].

In this paper, we focus on the split-step Fourier method. The efficiency of the split-step method depends on both the time-domain (or frequency-domain) resolution and on the distribution of step sizes along the fiber. In simulations of optical-fiber transmission systems, the time and frequency resolutions are determined by the bandwidth of the signal and the number of bits that are to be propagated through the system, respectively. Consequently, the properties of the signal determine the minimum required number of Fourier modes. Although the number of Fourier modes affects the accuracy of the numerical solution, as we will discuss later, it does not change the qualitative behavior of the spatial step-size selection algorithm. In this paper, we focus on the accuracy and efficiency of different spatial step-size selection criteria.

A variety of step-size selection criteria, most based on physical intuition, have been proposed for optimizing the split-step method. The figure of merit for each criterion is the computational cost for a given resulting global accuracy. Historically, in numerical methods used to solve (1), the step-size distribution was optimized for simulating soliton propagation. However, this optimization is not necessarily appropriate for modeling modern transmission systems, which often feature both high and low dispersion and relatively small nonlinearity, by which we mean that the nonlinear length scale is long compared to typical dispersion length scales.

The purpose of this paper is to implement an efficient systemindependent step-size selection criterion for solving the nonlinear Schrödinger equation that is based on bounding the local error, and to compare its performance to four commonly used step-size selection methods that are based on physical intuition. In the first of these four methods, called the nonlinear phase-rotation method, the step size is chosen so that the phase change 
due to nonlinearity does not exceed a certain limit. This method was designed with soliton propagation in mind. The second, the logarithmic step-size method, is designed to efficiently suppress spurious FWM, by employing a logarithmic distribution of the step sizes [6]. In the third method, the walk-off method, the step size is chosen to be inversely proportional to the product of the absolute value of dispersion and the spectral bandwidth of the signal. The idea behind this criterion is to resolve the collisions between pulses in different channels or at least to have a measure for the violation of this criterion. This method was designed for low power, multichannel systems. In the fourth, the constant step-size method, the step sizes are kept constant along the whole transmission path.

Finally, we implement a method we call the local-error method, in which the step size is selected by bounding the relative local error of the step. In addition, in this method, we obtain a higher order solution that is globally third-order accurate. The method is inspired by and closely related to widely used algorithms for adaptively controlling the step size in ordinary differential equation solvers [7]. In particular, we have adopted the well-known techniques of step-doubling to estimate the local error and linear extrapolation to obtain the higher order solution. To the best of our knowledge, although they are widely used in other fields, techniques such as these have not been previously used in simulations of optical-fiber transmission systems or even seriously investigated. As is typically the case for higher order schemes, our scheme has the advantage that it is much more computationally efficient than a second-order scheme when the global accuracy is high [7], [8]. On the other hand, it can be less efficient at low accuracy. This behavior is consistent with the results of Fornberg and Driscoll [9], who compared split-step methods of order 2, 4, and 6 with several higher order linear multistep methods. For a two-soliton collision, Fornberg and Driscoll showed that for the global error range of $10^{-3}-10^{-2}$, the second-order split-step scheme is more efficient than the fourth- and sixth-order schemes. However, for global errors smaller than $10^{-4}$, the higher order schemes become more efficient. We found similar qualitative behavior for the second-order schemes and third-order local-error method that we study here.

For typical realistic optical-fiber transmission systems, we will demonstrate that the walk-off method is the most efficient of the four methods in the range of accuracy of commercial interest. Nevertheless, the local-error method is still competitive in this accuracy range, and moreover, it is robust for a wide range of systems.

The remainder of the paper is organized as follows. First, we review the split-step method and the error associated with it. Then we describe the five implementations of the split-step method. Next, we discuss simulation results comparing the performance of these implementations. Finally, the conclusion follows.

\section{THEORY}

\section{A. Origin of the Split-Step Error}

To estimate the local and global errors in the split-step Fourier method it is convenient to represent (1) in the form

$$
\frac{\partial u(z, t)}{\partial z}=(\hat{D}+\hat{N}[u]) u(z, t)
$$

where $\hat{D}=-i\left(\beta^{\prime \prime} / 2\right) \partial^{2} / \partial t^{2}$ is the dispersion operator and $\hat{N}[u]=i \gamma|u|^{2}$ is the nonlinear operator. Although the following discussion is for the nonlinear Schrödinger equation (1), the arguments and conclusions also apply to the modified versions of (1) that model realistic optical-fiber transmission systems and to general reaction-diffusion equations. In the symmetric split-step scheme, the solution to (2) is approximated by

$u(z+h, t) \approx \exp \left(\frac{h}{2} \hat{D}\right) \exp \left\{h \hat{N}\left[u\left(z+\frac{h}{2}, t\right)\right]\right\} \exp \left(\frac{h}{2} \hat{D}\right)$.

Since the dispersion and nonlinear operators do not commute in general, the solution (3) is only an approximation to the exact solution. An argument based on the Baker-Campbell-Hausdorff formula shows that the local error, which is the error incurred in a single step of the symmetric split-step scheme, has a leadingorder term that is of third order in the step size $h$, i.e, the error is $O\left(h^{3}\right)$ [10]. When we state that an error is $O\left(h^{m}\right)$, we mean that it is bounded by $C h^{m}$ for some constant $C$. Since the total number of steps in a fiber span is inversely proportional to the average step size, the global error accumulated over a fiber span is second order in the step size $O\left(h^{2}\right)$.

Finding an optimal step-size distribution depends on the particular optical transmission system. We will review several criteria for choosing the step size in the split-step Fourier method and we will introduce a new criterion based on a measure of the local error.

\section{B. Nonlinear Phase-Rotation Method}

The nonlinear phase-rotation method is a variable step-size method that is designed for systems in which nonlinearity plays a major role. For a step of size $h$, the effect of the nonlinear operator $\hat{N}$ is to increment the phase of $u$ by an amount $\phi_{\mathrm{NL}}=$ $\gamma|u|^{2} h$. If we impose an upper limit $\phi_{\mathrm{NL}}^{\max }$ on the nonlinear phase increment $\phi_{\mathrm{NL}}$, we obtain the bound on the step size

$$
h \leq \frac{\phi_{\mathrm{NL}}^{\max }}{\gamma|u|^{2}} .
$$

This criterion for selecting the step size was originally applied to simulate soliton propagation and is widely used in optical-fiber transmission simulators. However, as we will show later, this approach is far from optimal for many modern communications systems.

\section{Spurious Four-Wave Mixing and Logarithmic Step-Size Distribution}

An improper distribution of the step sizes may lead not only to a general reduction of accuracy but also to numerical artifacts. Forghieri [11] demonstrates that the power of the four-wave mixing (FWM) products can be greatly overestimated by a constant step-size method, since FWM is a resonance effect. To efficiently suppress this numerical artifact, Bosco et al. [6] used a logarithmic distribution of the step sizes to keep the spurious FWM components below a certain level. For a fiber span of length $L$ and loss coefficient $\Gamma$, the step size of the $n$th step is given by

$$
h_{n}=-\frac{1}{2 \Gamma} \ln \left[\frac{1-n \sigma}{1-(n-1) \sigma}\right]
$$


where $\sigma=[1-\exp (-2 \Gamma L)] / K$ and $K$ is the number of steps per fiber span. We will call this implementation of the split-step method the logarithmic step-size method.

\section{Walk-Off Method}

In many optical-fiber communications systems, chromatic dispersion is the dominant effect and nonlinearity only plays a secondary role, particularly in multichannel systems in which the wavelength channels cover a broad spectrum. In this case, it can be reasonable to use the walk-off method, in which the step size is determined by the largest group velocity difference between channels. The basic idea is to choose the step size to be smaller than a characteristic walk-off length. In a multichannel system with large local dispersion, pulses in different channels move through each other very rapidly. To resolve the collisions between pulses in different channels, the step size in the walkoff method is chosen so that in a single step, two pulses in the two edge channels shift with respect to each other by a time that is a specified fraction of the pulse width. Consequently, the step size is given by

$$
h=\frac{C}{\Delta V_{g}}
$$

where $\Delta V_{g}$ is the largest group velocity difference between channels and $C$ is a constant that can vary from system to system. In any system, $\Delta V_{g}=\left|D_{2} \lambda_{2}-D_{1} \lambda_{1}\right|$, where $D_{1}$ and $D_{2}$ are the dispersions corresponding to the smallest and largest wavelengths $\lambda_{1}$ and $\lambda_{2}$. Since $\Delta V_{g}$ is constant in any particular kind of fiber, in a given type of fiber, the step size is constant. The walk-off method can be applied to single-channel as well as multichannel systems by choosing $\lambda_{1}$ and $\lambda_{2}$ at the two edges of the signal spectrum.

\section{E. Constant Step-Size Method}

The simplest way to implement the split-step Fourier method is to use a constant step size along the whole transmission path. The global accuracy can be improved only by increasing the total number of steps. Note that the walk-off and constant stepsize methods are identical in systems with only one type of fiber.

\section{F. Local-Error Method}

In practice, it is desirable to have a general criterion for choosing the step-size distribution that is close to optimal for an arbitrary system. Adaptive methods for controlling the step size using a measure of the local error are widely used in ordinary differential equation solvers [7]. We have implemented a scheme based on bounding the error in each step using the technique of step-doubling and local extrapolation. Given the field $u$ at a distance $z$, our aim is to compute the field at $z+2 h$. Suppose that we perform one step of size $2 h$ in a symmetric split-step scheme. We will refer to the solution obtained at $z+2 h$ as the coarse solution $u_{c}$. Since the local error in the symmetric split-step scheme is third order, there is a constant $\kappa$ so that

$$
u_{c}=u_{t}+\kappa(2 h)^{3}+O\left(h^{4}\right)
$$

where the true solution $u_{t}$ is the exact solution at $z+2 h$ obtained from the given solution at $z$. When we write that $u=v+O\left(h^{4}\right)$ for some functions $u$ and $v$, we mean that $|u-v|<C h^{4}$ for some constant $C$. Next, we return to $z$ and compute the fine solution $u_{f}$ at the same distance $z+2 h$ using two steps of size $h$. As done previously, the fine solution is related to the true solution by

$$
u_{f}=u_{t}+2 \kappa h^{3}+O\left(h^{4}\right) .
$$

By taking an appropriate linear combination of the fine and coarse solutions we can obtain an approximate solution at $z+2 h$ for which the leading order error term is of fourth order in the step size $h$ [7]. From (7) and (8), it follows that this higher order solution is given by

$$
u_{4}=\frac{4}{3} u_{f}-\frac{1}{3} u_{c}=u_{t}+O\left(h^{4}\right)
$$

which we take as the input to the next step of size $2 h$.

In the local-error method, the step size is adaptively chosen so that the local error incurred from $z$ to $z+2 h$ is bounded within a specified range. Now the relative local error $\delta_{4}$ of the higher order solution is defined by

$$
\delta_{4}=\frac{\left\|u_{4}-u_{t}\right\|}{\left\|u_{t}\right\|}
$$

where the norm $\|u\|$ is defined as $\|u\|=\left(\int|u(t)|^{2} d t\right)^{1 / 2}$. However, since we cannot compute the true solution $u_{t}$ in practice, we cannot compute the local error using (10). Instead, we define the relative local error of a step to be the local error in the coarse solution relative to the fine solution

$$
\delta=\frac{\left\|u_{f}-u_{c}\right\|}{\left\|u_{f}\right\|} .
$$

Notice that $\delta$ is a measure of the true local error $\delta_{4}$ since $\delta$ can be obtained from $3 \delta_{4}$ by replacing $u_{t}$ by $u_{f}$. The step size is chosen by keeping the relative local error $\delta$ within a specified range $\left(1 / 2 \delta_{G}, \delta_{G}\right)$, where $\delta_{G}$ is the goal local error. If $\delta>2 \delta_{G}$, the solution is discarded and the step size is halved. If $\delta$ is in the range $\left(\delta_{G}, 2 \delta_{G}\right)$, the step size is divided by a factor of $2^{1 / 3}$ for the next step. If $\delta<1 / 2 \delta_{G}$, the step size is multiplied by a factor of $2^{1 / 3}$ for the next step.

Rather than simply computing the fine solution, our method computes both the fine and coarse solutions. Although it requires 50\% more Fourier transforms than does the standard symmetric split-step method, the method yields both a higher order solution, which is globally third-order accurate, and a measure of the relative local error that is used to control the step size. However, it is important to understand that the higher order solution $u_{4}$ is not always more accurate than the fine solution $u_{f}$, especially when the step size is large, since we are bounding the local error $\delta$ of the coarse solution relative to the fine solution, rather than the true local error $\delta_{4}$ of the scheme.

Since we do not make any assumptions about the physical properties of the system, such as the amount of nonlinearity or dispersion, we expect the local-error method to work well in an arbitrary system. In order to simulate a system with optimal efficiency, one first needs to investigate it to ascertain the major sources of the split-step error. Assuming that the system is dominated by one source of error, one can select an appropriate criterion for choosing the step sizes. The local-error method allows us to deal with general systems when the major source of error is unknown or may even change during the propagation, 

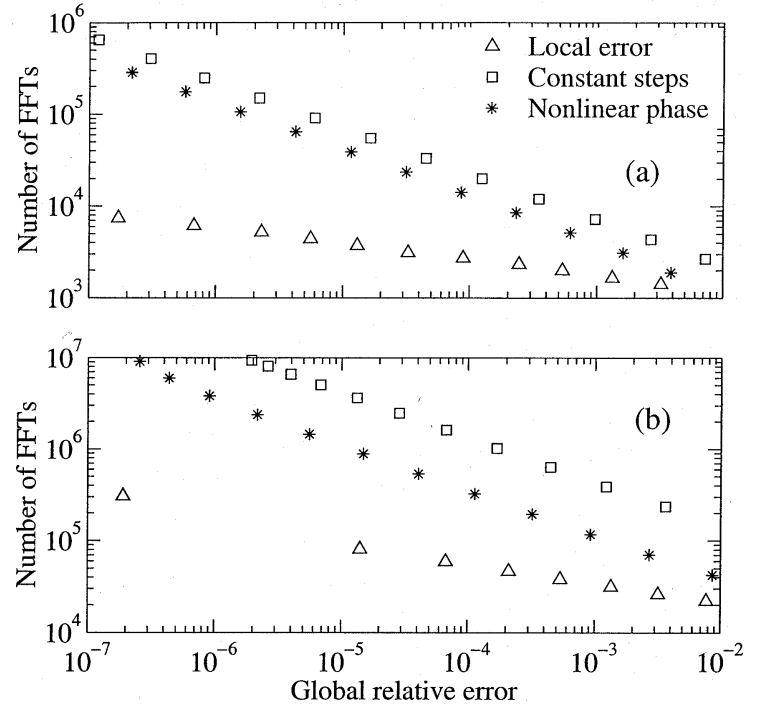

Fig. 1. Plot of the total number of FFTs versus global relative error $\varepsilon$ for (a) second-order and (b) fifth-order solitons.

or when performing a series of simulations in which the system parameters are varied. The method can be applied to a variety of systems without sacrificing too much computational efficiency.

\section{NUMERICAL RESULTS}

In this section, we compare the efficiency of the five implementations of the split-step method described in Section II. Since most of the computational time is consumed by evaluating fast Fourier transforms (FFTs), we use the number of FFTs per simulation as a measure of the total computational cost [9]. We used the following scheme to compare the different methods. First, we compute a solution $u_{a}$ that is accurate to machine precision using the standard symmetric split-step method (with step sizes on the order of $5 \mathrm{~cm}$ ). Next, we compute the numerical solution $u_{n}$ for each of the different split-step implementations, and calculate the global relative error $\varepsilon$ defined by

$$
\varepsilon=\frac{\left\|u_{n}-u_{a}\right\|}{\left\|u_{a}\right\|}
$$

where we use the norm defined in Section II-F. We compare the performance of the different methods by plotting the number of FFTs versus the global relative error.

\section{A. Higher Order Solitons}

We start with the propagation of second-order and fifth-order solitons. These systems are both highly nonlinear. In addition, higher order solitons are very sensitive to numerical errors, thus requiring an efficient adaptive algorithm. The exact functional form of the $N$-soliton solution can be found in [3], [5]. We use an anomalous-dispersion fiber with $\beta^{\prime \prime}=-0.1 \mathrm{ps}^{2} / \mathrm{km}$. The initial pulse is a hyperbolic secant of the form $u(t)=$ $A \eta\left(\left|\beta^{\prime \prime}\right| / \gamma\right)^{1 / 2} \operatorname{sech}(\eta t)$, where the nonlinear coefficient is $\gamma=$ $2.2 \mathrm{~W}^{-1} \mathrm{~km}^{-1}$, the inverse pulse duration is $\eta=0.44 \mathrm{ps}^{-1}$, and where $A=2$ and $A=5$ for the second-order and fifth-order solitons, respectively. The corresponding FWHM pulse duration is $4 \mathrm{ps}$, and the peak powers are 35 and $220 \mathrm{~mW}$ for the

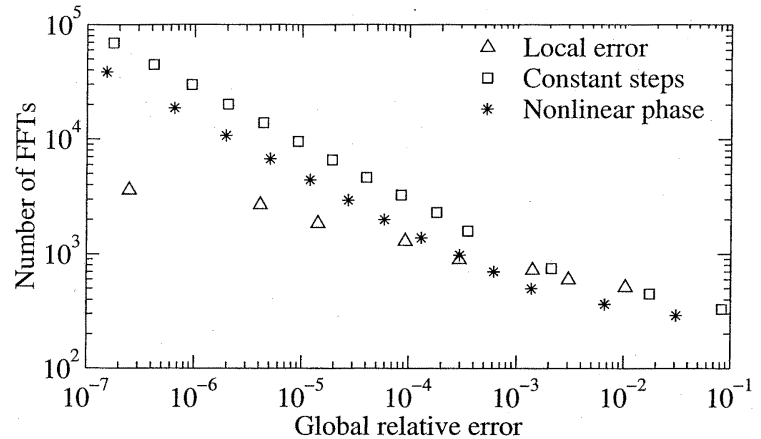

Fig. 2. Plot of the total number of FFTs versus global relative error $\varepsilon$ for a collision of two first-order solitons.

second-order and fifth-order solitons, respectively. The number of Fourier modes is 1024 and the simulation time window is $50 \mathrm{ps}$. We show the performance of the different implementations of the split-step method applied to the second-order soliton in Fig. 1(a) and to the fifth-order soliton in Fig. 1(b). In Fig. 1, we have plotted the number of FFTs versus the global relative error for the different step-size criteria. Although the performance of the local-error method is not significantly better in the range of low accuracy values $10^{-2}-10^{-3}$ at high accuracy, the computational cost of the local-error method is one or two orders of magnitude less than for other methods. Notice that the nonlinear phase method performs better than the constant step-size method, consistent with the system's large nonlinearity. The slope of the local-error method curve is less than those of the other two methods since the constant step size and nonlinear phase methods are globally second-order accurate, while the local-error method is globally third-order accurate. The walk-off and constant step-size methods are identical since this system includes only one type of fiber. The logarithmic step-size method reduces to the constant step-size method because the fiber is lossless and (5) leads to a constant step-size distribution.

\section{B. Soliton Collisions}

Soliton collisions can be a good test for numerical methods because the subtle effect of FWM cancellation after the collision is very sensitive to numerical errors [3]. The fiber type and the initial pulse shape are the same as in Section III-A, except that $A=1$. The pulse duration is $4 \mathrm{ps}$ and the peak power is $8.8 \mathrm{~mW}$. We launch two soliton pulses separated in time by $100 \mathrm{ps}$ and with a central-frequency difference of $800 \mathrm{GHz}$. The number of Fourier modes is 3072 and the simulation time window is 400 ps. We show the performance of the different methods in Fig. 2. The local error, constant step size, and nonlinear phase-rotation methods perform equally well at low accuracy when the global error is in the range $10^{-3}-10^{-1}$, while the local-error method is much more efficient when the global error is less than $10^{-4}$. Global errors less than $10^{-4}$ are required to estimate the FWM terms correctly and to have them cancel out after the collision. The nonlinear phase method still works better than the constant step-size method because the nonlinear interactions are critical in the propagation. As in Section III-A, the logarithmic step size and walk-off methods reduce to the constant step-size method. 


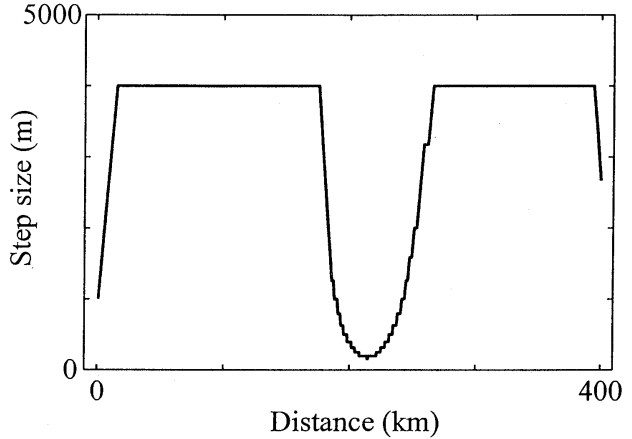

Fig. 3. Step size $h$ as a function of distance for the local-error method applied to a collision of two first-order solitons.

Using the example of a soliton collision, we illustrate the adaptive behavior of the local-error algorithm. Fig. 3 shows the step size as a function of propagation distance for the soliton collision when the targeted range for the local error is $\left(0.5 \times 10^{-5}\right.$, $\left.10^{-5}\right)$, and the initial guess for the step size is $1000 \mathrm{~m}$. Since the local error for this initial step is much less than the targeted range of values, at each step, the step sizes are increased until the local error is within the targeted range. The pulse collision occurs at a distance of $200 \mathrm{~km}$. At this point, we observe a significant decrease in the step size, which is necessary to accurately resolve the collision. After the collision, the step size is increased to the same value as before the collision. The last step is smaller than the previous step simply because the remaining section of the fiber is shorter than the step size chosen by the algorithm.

\section{Single-Channel Systems}

In this section, we study periodically stationary dispersionmanaged soliton (DMS) and chirped-return-to-zero (CRZ) systems that resemble experimental systems [12], [13]. The DMS system is highly nonlinear, meaning that both dispersion and nonlinearity determine the signal evolution, while the CRZ system is quasilinear and the evolution is mostly determined by dispersion [14], [15]. Thus we are studying the four split-step implementations using two different types of systems. We include fiber attenuation and gain, but we do not consider amplifier noise. We use random bit strings of length 64 that repeat periodically. We stress that our goal is to test the performance of the numerical methods for realistic systems rather than to achieve optimal propagation. Consequently, it is important that we have pulse streams rather than single pulses, that we use dispersion management, and that we include the effects of fiber loss and amplifier gain.

The DMS system is based on a 107-km dispersion map, which consists of four dispersion-shifted fiber spans, each of $25 \mathrm{~km}$, with normal dispersion equal to $-1.10 \mathrm{ps} / \mathrm{nm} \cdot \mathrm{km}$, followed by $7 \mathrm{~km}$ of standard single-mode fiber with anomalous dispersion of $16.6 \mathrm{ps} / \mathrm{nm} \cdot \mathrm{km}$ at $1551 \mathrm{~nm}$ [12]. The loss in both fibers is 0.21 $\mathrm{dB} / \mathrm{km}$, and the amplifier spacing is $25 \mathrm{~km}$ with an additional amplifier after the standard single-mode fiber. We use Gaussian pulses with a FWHM duration of $9 \mathrm{ps}$, as is appropriate for a 10 $\mathrm{Gb} / \mathrm{s}$ bit rate. The peak power is $8 \mathrm{~mW}$. The signal is launched in the middle of a span of anomalous fiber to ensure the peri-
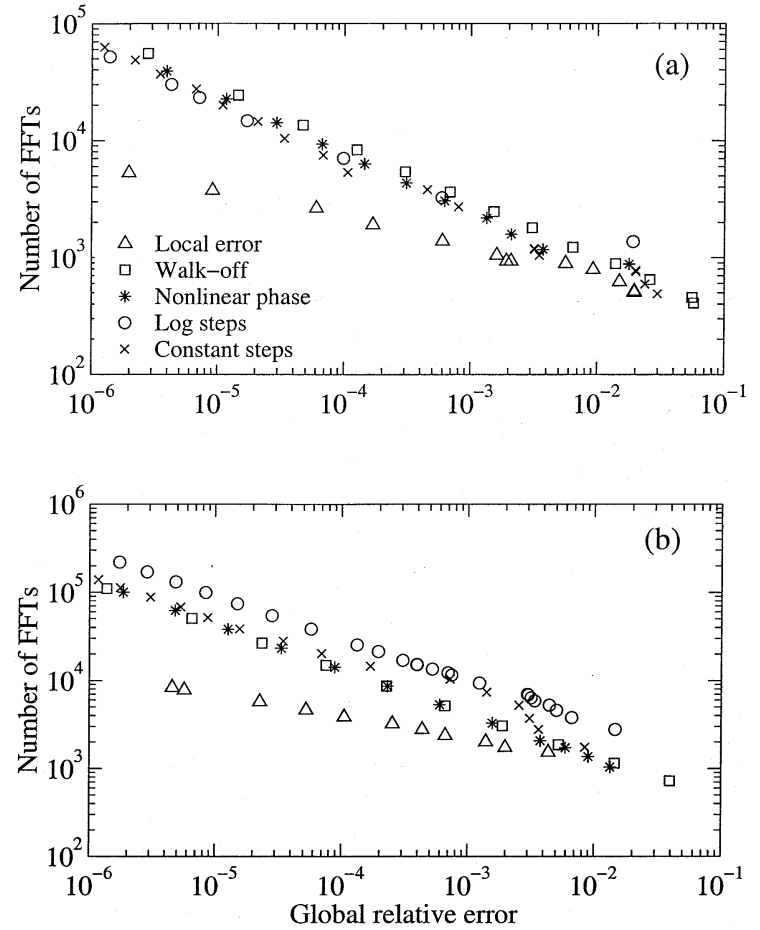

Fig. 4. Plot of the total number of FFTs versus global relative error $\varepsilon$ for the single-channel (a) DMS and (b) CRZ systems.

odicity of the pulse shape as it propagates along the fiber. The propagation distance is $1280 \mathrm{~km}$. The simulation time window is $6400 \mathrm{ps}$ and the number of Fourier modes is 6144 . We have not included a dispersion slope in this system since there is only a single channel and previous work indicates that higher order dispersion plays no role [12].

The CRZ system is based on a $180 \mathrm{~km}$ dispersion map consisting of $160 \mathrm{~km}$ of dispersion-shifted fiber with dispersion $-2.44 \mathrm{ps} / \mathrm{nm} \cdot \mathrm{km}$ followed by $20 \mathrm{~km}$ of standard fiber with dispersion $16.55 \mathrm{ps} / \mathrm{nm} \cdot \mathrm{km}$ [13]. The dispersion slope is $0.075 \mathrm{ps}^{2} / \mathrm{nm} \cdot \mathrm{km}$ and the fiber loss is $0.21 \mathrm{~dB} / \mathrm{km}$ for both fibers, while the amplifier spacing is $45 \mathrm{~km}$. Symmetric dispersion pre- and postcompensation is performed using fiber spans of length $2.0 \mathrm{~km}$, where the dispersion is $93.5 \mathrm{ps} / \mathrm{nm} \cdot \mathrm{km}$, the slope is $-0.2 \mathrm{ps}^{2} \mathrm{~nm} \cdot \mathrm{km}$ and the loss is $0.5 \mathrm{~dB} / \mathrm{km}$. The initial pulses are phase-modulated, raised-cosine pulses with 1-mW peak power and a chirp parameter equal to -0.6 [14]. The bit rate is $10 \mathrm{~Gb} / \mathrm{s}$ and the propagation distance is 1800 $\mathrm{km}$. The simulation time window is $6400 \mathrm{ps}$ and the number of Fourier modes is 4096.

The performance of the four split-step implementations for the single-channel DMS and CRZ systems is shown in Fig. 4(a) and (b), respectively. In both systems, the local-error method performs best over the entire range. Due to its higher order of accuracy, the data points for the local-error method lie on a line with a smaller absolute slope than those of the other methods, as expected. However, all methods become comparable in the range of global errors $10^{-3}-10^{-1}$, the region of most interest in simulating fiber-optic links. We note however, that in the CRZ system the performance of the logarithmic step-size method is somewhat poorer than that of the nonlinear phase and walk-off methods. 


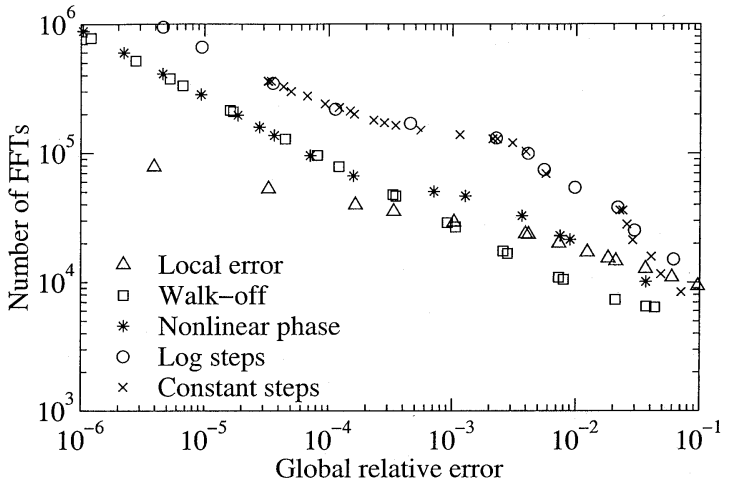

Fig. 5. Plot of the total number of FFTs versus global relative error $\varepsilon$ for the multichannel CRZ system.

\section{Multichannel CRZ System}

In order to compare the split-step implementations for modeling multichannel communications systems, we used the same CRZ system as described in Section III-C. In Fig. 5, we show the performance of the split-step selection criteria on a five-channel CRZ system with a 50-GHz channel spacing. As in the singlechannel case, the local-error method is much more efficient at high accuracy. However, at low accuracy, with the global error in the range $10^{-3}-10^{-1}$, which is typical for most practical systems, the walk-off method performs best. At low accuracy, the local-error method does not perform as well as the walk-off method for the following reasons. First, in the multichannel CRZ system, the step size within each fiber in the local-error method varies approximately within a factor of two, and the average value is comparable to the step size in the walk-off method for a given global error. However, each pair of steps in the local-error method is 50\% more expensive than in the walk-off method. In addition, when the step size is large and the global accuracy is low, the higher order solution $u_{4}$ may not be as accurate as the fine solution $u_{f}$. Indeed, we have observed that the local-error method performs slightly better at low global accuracy if we keep the fine solution $u_{f}$ instead of the higher order solution $u_{4}$ at each step.

Next, we observe that the nonlinear phase-rotation method does not perform as well as the walk-off method in the multichannel CRZ system, although the performance of the two methods is comparable in the single-channel DMS and CRZ systems. There are two major reasons for this behavior. First, in contrast to the single-channel case, the walk-off criterion becomes more physically relevant in a WDM system, in which pulses in different channels collide. Second, the step size in the nonlinear phase-rotation method is determined by the peak power in the time domain. In the single-channel CRZ system, the power function contains spikes due to the overlap between neighboring pulses. However, between amplifiers the peak power decreases monotonically with distance due to fiber attenuation. By contrast, the peak power of the multichannel system does not decrease monotonically with distance but contains irregular spikes because pulses from different channels rapidly pass through each other. As a consequence, there is a significant proportion of step sizes in the nonlinear phase-rotation method that are much smaller than they need to be for a given global

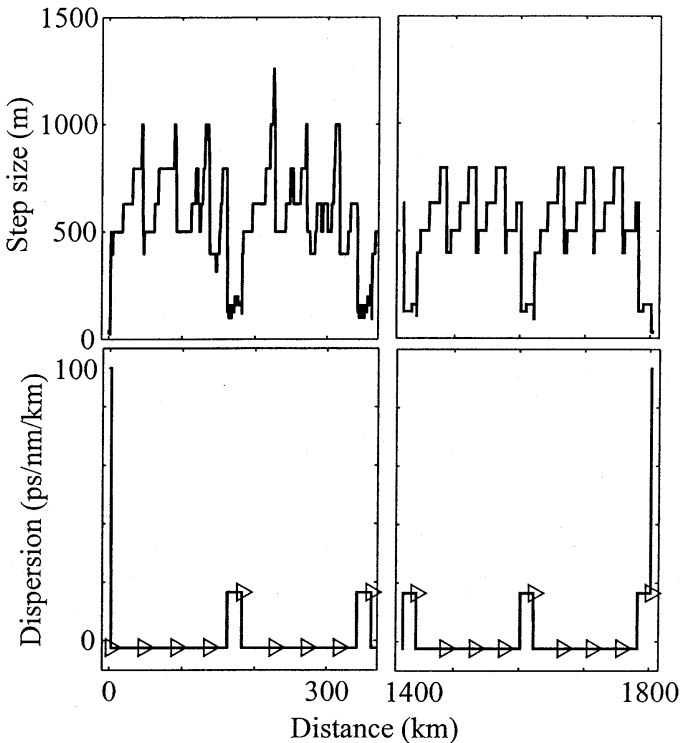

Fig. 6. Step size $h$ as a function of distance for the local-error method applied to the multichannel CRZ system. The upper two plots show the step sizes for the first two and last two periods of the dispersion map, and the lower two plots show the corresponding portions of the dispersion map. Triangles indicate the positions of amplifiers.

accuracy. The logarithmic step-size method is not efficient in the CRZ system because the step-size choice is only based on limiting spurious FWM, which is only one of the potential sources of error in a multichannel simulation. We also found that in the logarithmic step-size method, the error grows most rapidly in fibers with high dispersion. We find that the constant step-size method is inefficient in the multichannel CRZ system. The reason it performs so poorly is that for a given step size the global error does not accumulate linearly with distance. Consequently, in some sections of the transmission line the global error grows rapidly, while in others the error accumulates very slowly and computational effort is wasted.

In Fig. 6, we show the step sizes in the local-error method as a function of propagation distance when the targeted range for the local error is $\left(0.5 \times 10^{-4}, 2 \times 10^{-4}\right)$. The upper two plots show the step sizes for the first two and last two periods of the dispersion map, and the lower two plots show the corresponding portions of the dispersion map. The amplifiers, marked by triangles, are placed after the precompensation fiber and then every $45 \mathrm{~km}$. Notice that the step size increases as the signal power and the strength of the nonlinear interactions decrease due to the fiber loss. Also note that step size is smaller in fibers with higher dispersion since the pulses in neighboring channels move faster with respect to each other.

To characterize the dependence of the local-error method on the number of Fourier modes, we modeled the multichannel CRZ system with different numbers of Fourier modes $N$. The results in Fig. 5 were obtained with $N=4096$. We also simulated the system with $N=3072, N=6144$, and $N=8192$. In each case, the log-log plots of the number of FFTs versus the global error have the same slope as for the local-error method plot shown in Fig. 5. However, the curves are slightly shifted with respect to each other. The number of FFTs required to achieve a given global error can increase by a factor of two as $N$ is increased from 3072 to 6144 . Since we keep the time 

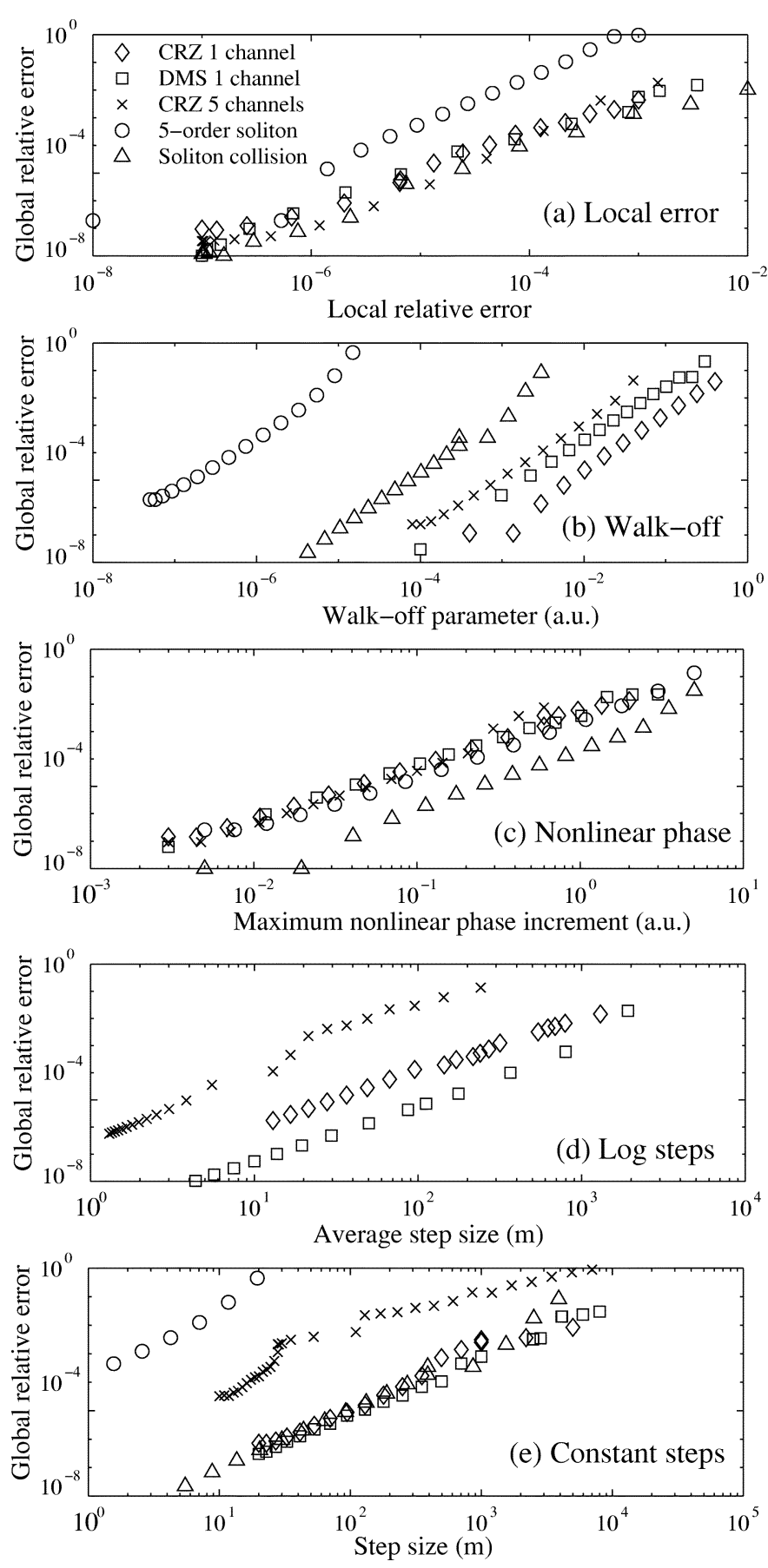

Fig. 7. Plot of the global error as a function of method parameter for (a) local error, (b) walk-off, (c) nonlinear phase, (d) logarithmic step, and (e) constant step methods.

window fixed, the frequency window increases as $N$ increases, and more high-frequency components contribute to the global error. However, if we further increase $N$ from 4096 to 8192 , the number of FFTs grows by less than $30 \%$, since the additional high frequency modes lie well outside the bandwidth of the WDM signal and contribute little to the error.

\section{E. Variation of Method Parameters}

In this section, we address two important questions concerning how the method parameter should be chosen to achieve a desired global accuracy. The method parameter is the parameter in a split-step method that we vary to adjust the accuracy of the method. First, for a given global error, how much does the method parameter depend on the particular system? Second, by what factor should the method parameter be decreased to halve the global error?

To answer the first question, in Fig. 7(a)-(e), we show the dependence of the global error on the method parameter for the local error, walk-off, nonlinear phase, logarithmic step, and constant step-size methods, respectively. Although the walk-off method is the most efficient in some cases, it exhibits the worst system dependence. In particular, for the five systems we studied, when the global error is $10^{-3}$, the walk-off parameter varies over five orders of magnitude, whereas the parameter for the other three methods vary only over one to two orders of magnitude. Even omitting the two soliton systems from the comparison, the walk-off method has a greater system dependence than the local-error method. Consequently, each new system requires a significantly different walk-off parameter to achieve the same global accuracy.

To answer the second question, we examine the slopes of the curves in Fig. 7. For the walk-off, nonlinear phase, and logarithmic steps methods, the slopes are approximately 2 , as expected, since these three schemes are second order and the step sizes depend linearly on the method parameter. Ideally, the global error should depend linearly on the local error. However, for the local-error method, the slopes of the curves in Fig. 7(a) are approximately 1.3 rather than 1 . The reason for this discrepancy is that the true local error (10) is unavailable. Instead, we use an estimate of local error given by (11). In addition, in our local-error algorithm, the local error (11) is maintained within a range of values rather than being kept constant.

\section{CONCLUSION}

We have studied the performance of different implementations of the symmetric split-step Fourier method for solving the nonlinear Schrödinger equation applied to various optical-fiber transmission systems. We developed an implementation of the symmetric split-step Fourier method that is globally third-order accurate and for which the step sizes are chosen using a criterion that keeps the local error within a specified range. We showed that the local-error method performs best for modeling optical solitons, soliton-pulse interactions, and single-channel transmission systems. Because it is a higher order method, the local-error method is much more computationally efficient at high accuracy than the other three methods we considered for all of the systems we studied. This behavior is expected with higher order schemes [9]. Moreover, even at low accuracy, the local-error method has the advantage that it is robust for arbitrary systems.

We find that the nonlinear phase-rotation method is inefficient for modeling typical modern optical-fiber transmission systems, although it performs reasonably well for solitons. The logarithmic step-size method, which is based on bounding the spurious FWM in each step [6], and the constant step-size method are not efficient for multichannel systems, although they can 
be as efficient as the nonlinear phase and walk-off methods in single-channel systems. Finally, the walk-off method, in which the step size is chosen to be inversely proportional to the fiber dispersion, performs well for multichannel systems over the accuracy range of interest in commercial applications.

\section{ACKNOWLEDGMENT}

The authors would like to thank B. Marks for many illuminating conversations and helpful comments.

\section{REFERENCES}

[1] A. Hasegawa and F. Tappert, "Transmission of stationary nonlinear optical pulses in dispersive dielectric fibers I. Anomalous dispersion," Appl. Phys. Lett., vol. 23, pp. 142-144, 1973.

[2] G. P. Agrawal, Nonlinear Fiber Optics, 2nd ed. London, U.K.: Academic, 1995.

[3] A. Hasegawa and Y. Kodama, Solitons in Optical Communications. Oxford, U.K.: Clarendon, 1995.

[4] C. R. Menyuk, "Application of multiple-length-scale methods to the study of optical fiber fiber transmission," J. Eng. Math., vol. 36, pp. 113-136, 1999.

[5] V. E. Zakharov and A. B. Shabat, "Exact theory of two-dimensional selffocusing and one-dimensional self-modulation of waves in nonlinear media," Sov. Phys.-JETP, vol. 34, pp. 62-69, 1972.

[6] G. Bosco, A. Carena, V. Curri, R. Gaudino, P. Poggiolini, and S. Benedetto, "Suppression of spurious tones induced by the split-step method in fiber systems simulation," IEEE Photon. Technol. Lett., vol. 12, pp. 489-491, May 2000.

[7] C. W. Gear, Numerical Initial Value Problems in Ordinary Differential Equations. NJ: Prentice-Hall, 1971.

[8] K. J. Blow and D. Wood, "Theoretical description of transient stimulated Raman scattering in optical fibers," IEEE J. Quantum Electron., vol. 25, pp. 2665-2673, Dec. 1989

[9] B. Fornberg and T. A. Driscoll, "A fast spectral algorithm for nonlinear wave equations with linear dispersion," J. Comp. Phys., vol. 155, pp. 456-467, 1999

[10] J. A. Fleck, J. R. Morris, and M. D. Feit, "Time dependent propagation of high energy laser beams through the atmosphere," Appl. Phys., vol. 10, pp. 129-160, 1976.

[11] F. Forghieri, "Modeling of wavelength multiplexed lightwave systems," in Proc. OFC, Dallas, TX, Feb. 1997.

[12] R.-M. Mu, V. S. Grigoryan, C. R. Menyuk, G. M. Carter, and J. M. Jacob, "Comparison of theory and experiment for dispersion-managed solitons in a recirculating fiber loop," IEEE J. Select. Topics Quantum Electron., vol. 6, pp. 248-257, Mar./Apr. 2000.

[13] N. S. Bergano, C. R. Davidson, N. A. Mills, P. Corbert, S. G. Evangelides, B. Pedersen, R. Menges, J. L. Zyskind, J. W. Sulhoff, A. K. Srivastava, C. Wolf, and J. Judkins, "Long-haul WDM transmission using optimal channel modulation," in Proc. OFC, Dallas, TX, 1997.

[14] R.-M. Mu and C. R. Menyuk, "Convergence of the chirped return-to-zero and soliton modulation formats in WDM systems," $J$. Lightwave Technol., vol. 20, pp. 608-617, 2002.

[15] C. R. Menyuk, G. M. Carter, W. L. Kath, and R.-M. Mu, "Dispersionmanaged solitons and chirped return to zero: What is the difference?," in Optical Fiber Telecommunications, I. P. Kaminow and T. Li, Eds. San Diego, CA: Academic, 2002, ch. 7, pp. 305-328.

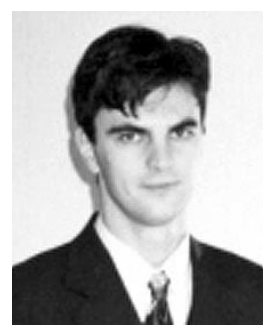

Oleg V. Sinkin (M'00) was born in Protvino, Russia, in 1976. He received the M.S. degree in applied physics and mathematics from the Moscow Institute of Physics and Technology, Moscow, Russia, in 1999. He is currently working toward the Ph.D. degree in electrical engineering at the University of Maryland Baltimore County.

His research areas have included fiber lasers and amplifiers and modeling optical fiber communications systems.

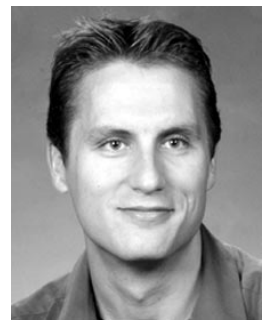

Ronald Holzlöhner (S'00) was born in Essen, Germany. He received the M.S. degree in physics from the Technical University of Berlin, Berlin, Germany, in 1998. He studied at the University of California, Santa Barbara, in 1995 to 1996 as a Fulbright exchange student. He is currently working toward the $\mathrm{Ph}$.D. degree at the University of Maryland Baltimore County (UMBC).

While at UMBC, he worked as a part-time consultant for VPIsystems, Inc.

Mr. Holzlöhner won the IEEE LEOS Student Fel-

lowship Award in 2000.

John Zweck received the Ph.D. degree in mathematics from Rice University, Houston, TX, in 1993.

He is a Research Associate Professor in the Department of Computer Science and Electrical Engineering at the University of Maryland Baltimore County. He has done research in differential geometry, human and computer vision, and the modeling of optical fiber communications systems.

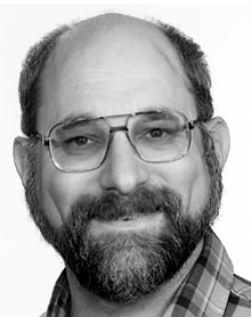

Curtis R. Menyuk (SM'88-F'98) was born on March 26, 1954. He received the B.S. and M.S. degrees from the Massachusetts Institute of Technology (MIT), Cambridge, in 1976 and the Ph.D. degree from the University of California, Los Angeles (UCLA), in 1981.

$\mathrm{He}$ has worked as a Research Associate at the University of Maryland, College Park, and at Science Applications International Corporation in McLean, VA. In 1986, he became Founding Member and an Associate Professor in the Department of Electrical Engineering at the University of Maryland Baltimore County (UMBC). In 1993, he was promoted to Professor. He was on partial leave from UMBC from Fall 1996 until Fall 2002. From 1996 to 2001, he worked part-time for the Department of Defense (DoD), codirecting the Optical Networking program at the DoD Laboratory for Telecommunications Sciences in Adelphi, MD, from 1999 to 2001. From 2001 to 2002, he was Chief Scientist at PhotonEx Corporation, Maynard, MA. He has authored or coauthored more than 160 archival journal publications as well as numerous other publications and presentations. He has also edited two books. For the last 15 years, his primary research area has been theoretical and computational studies of fiber-optic communications. The equations and algorithms that he and his research group at UMBC have developed to model optical fiber transmission systems are used extensively in the telecommunications industry.

Dr. Menyuk is a Member of the Society for Industrial and Applied Mathematics and the American Physical Society and is a Fellow of the Optical Society of America. He is a former UMBC Presidential Research Professor. 